Chronic Obstructive Pulmonary Diseases:

Journal of the COPD Foundation

\author{
Original Research
}

\title{
Nutrition and Markers of Disease Severity in Patients With Bronchiectasis
}

Katherine A. Despotes, $\mathrm{MD}^{1}$ Radmila Choate, $\mathrm{PhD}, \mathrm{MPH}^{2,3}$ Doreen Addrizzo-Harris, $\mathrm{MD}^{4}$

Timothy R. Aksamit, MD ${ }^{5}$ Alan Barker, MD ${ }^{6}$ Ashwin Basavaraj, MD ${ }^{4}$ Charles L. Daley, MD 7

Edward Eden, $\mathrm{MD}^{8}$ Angela DiMango, MD ${ }^{9}$ Kevin Fennelly, MD ${ }^{10}$ Julie Philley, MD ${ }^{11}$

Margaret M. Johnson, MD ${ }^{12}$ Pamela J. McShane, MD ${ }^{13}$ Mark L. Metersky, MD ${ }^{14}$ Anne E. O’Donnell, MD $^{15}$

Kenneth N. Olivier, MD, MPH ${ }^{10}$ Matthias A. Salathe, MD ${ }^{16}$ Andreas Schmid, MD ${ }^{16}$ Byron Thomashow, MD 9

Gregory Tino, MD ${ }^{17}$ Kevin L. Winthrop, MD, MPH ${ }^{6,18}$ Michael R. Knowles, MD ${ }^{1}$

Mary Leigh Anne Daniels, MD, MPH ${ }^{1}$ Peadar G. Noone, MD $^{1}$

\section{Abstract}

Background: Increasing numbers of patients are being diagnosed with bronchiectasis, yet much remains to be elucidated about this heterogeneous patient population. We sought to determine the relationship between nutrition and health outcomes in non-cystic fibrosis (non-CF) bronchiectasis, using data from the U.S. Bronchiectasis Nontuberculous Mycobacterial Research Registry (U.S. BRR).

Methods: This was a retrospective, observational, longitudinal study using 5-year follow-up data from the BRR. Bronchiectasis was confirmed on computed tomography (CT). We stratified patients into nutrition categories using body mass index (BMI), and correlated BMI to markers of disease severity.

Results: Overall, $\mathrm{n}=496$ patients (mean age $64.6 \pm 13$ years; 83.3\% female) were included. At baseline $12.3 \%(\mathrm{n}=61)$ were underweight $\left(\mathrm{BMI}<18.5 \mathrm{~kg} / \mathrm{m}^{2}\right), 63.9 \%(\mathrm{n}=317)$ had normal weight $\left(\mathrm{BMI} \geq 18.5 \mathrm{~kg} / \mathrm{m}^{2}\right.$ and $\left.<25.0 \mathrm{~kg} / \mathrm{m}^{2}\right), 17.3 \%(\mathrm{n}=86)$ were overweight $\left(\mathrm{BMI} \geq 25.0 \mathrm{~kg} / \mathrm{m}^{2}\right.$ and $\left.<30.0 \mathrm{~kg} / \mathrm{m}^{2}\right)$, and $6.5 \%(\mathrm{n}=32)$ were obese $\left(\mathrm{BMI} \geq 30 \mathrm{~kg} / \mathrm{m}^{2}\right)$. Men were overrepresented in the overweight and obese groups $(25.6 \%$ and $43.8 \%$ respectively, $p<0.0001$ ). Underweight patients had lower lung function (forced expiratory volume in 1 second $\left[\mathrm{FEV}_{1}\right] \%$ predicted) than the other weight groups (64.5 \pm 22 , versus $73.5 \pm 21,68.5 \pm 20$, and $76.5 \pm 21$ in normal, overweight, and obese groups respectively, $p=0.02$ ). No significant differences were noted between BMI groups for other markers of disease severity at baseline, including exacerbation frequency or hospitalization rates. No significant differences were noted in BMI distribution between patients with and without Pseudomonas, nontuberculous mycobacteria, or by cause of bronchiectasis. The majority of patients demonstrated stable BMI over 5 years.

Conclusions: Although underweight patients with bronchiectasis have lower lung function, lower BMI does not appear to relate to other markers of disease severity in this patient population.

\footnotetext{
Abbreviations: non-cystic fibrosis, non-CF; U.S. Bronchiectasis Research Registry, U.S. BRR; computed tomography, CT, body mass index, BMI; forced expiratory volume in 1 second, FEV $_{\mathbf{1}}$; Bronchiectasis Severity Index, BSI; nontuberculous Mycobacterial, NTM; chronic obstructive pulmonary disease, COPD; gastroesophageal reflux disease, GERD; Pseudomonas aeruginosa, P. aeruginosa; inflammatory bowel disease, IBD; forced vital capacity, FVC; Mycobacterium avium complex, MAC; standard deviation, SD; analysis of variance, ANOVA; primary ciliary dyskinesia, PCD; alpha-1 antitrypsin deficiency, AATD

Funding Support: Not applicable.

Date of Acceptance: June 30, 2020

Citation: Despotes KA, Choate R, Addrizzo-Harris D, et al. Nutrition and markers of disease severity in patients with bronchiectasis. Chronic Obstr Pulm Dis. 2020;7(4):390-403. doi: https://doi.org/10.15326/jcopdf.7.4.2020.0178
} 
1 Department of Medicine, University of North Carolina at Chapel Hill

2 Research, COPD Foundation, Washington, DC

3 College of Public Health, University of Kentucky, Lexington

4 School of Medicine, New York University, New York, New York

5 Pulmonary Disease and Critical Care Medicine, Mayo Clinic, Rochester, Minnesota

6 Department of Pulmonary and Critical Care, School of Medicine, Oregon Health and Science University, Portland

7 Division of Mycobacterial and Respiratory Infections, National Jewish Health, Denver, Colorado

8 Icahn School of Medicine, Mt. Sinai West and Mt. Sinai St Luke's Hospitals, Mt. Sinai, New York

9 Center for Chest Disease, Columbia College of Physicians and Surgeons, New York, New York

10 National Heart, Lung, and Blood Institute, National Institutes of Health, Bethesda, Maryland

11 University of Texas at Tyler

12 Center for Chest Disease, Mayo Clinic Florida, Jacksonville

13 Department of Medicine, University of Chicago, Chicago, Illinois

14 Division of Pulmonary and Critical Care Medicine, School of Medicine, University of Connecticut, Farmington

15 Georgetown University Medical Center, Washington, DC

16 University of Kansas Medical Center, Kansas City

17 Perelman School of Medicine, University of Pennsylvania, Philadelphia

18 Department of Infectious Disease, School of Medicine, Oregon Health and Science University School of Medicine, Portland

Address correspondence to:

Katherine A Despotes, MD

Phone: (919)649-0614

Email: katherine.despotes@unchealth.unc.edu

\section{Keywords:}

bronchiectasis; nutrition; BMI; outcomes; U.S. Bronchiectasis Research Registry

\section{This article contains an online supplement.}

\section{Introduction}

Bronchiectasis is a clinical syndrome characterized by chronic cough, usually productive of sputum, punctuated by recurrent respiratory infections or exacerbations, and marked by thickened, dilated airways on computed tomography (CT) scan. ${ }^{1,2}$ It frequently has a significant impact on patients' quality of life. ${ }^{3-5}$ Awareness of the disease has increased recently, as have incidence and prevalence. ${ }^{6-9}$ This is likely in part due to better radiographic techniques to recognize and diagnose bronchiectasis as well as improved surveillance. ${ }^{3,10}$ Recent data suggests that more patients are being diagnosed with bronchiectasis; between 340,000 and 520,000 are estimated to have been diagnosed in the United States alone. ${ }^{1,3}$ One particular challenge is that the patient population is very heterogeneous by geographic region, across age groups, with regards to underlying etiologies, and in sex predominance. ${ }^{1,9,11-13}$ As a result, clinical trials of novel therapies have proven difficult. ${ }^{3,14-19}$ It is important, therefore, to characterize sub-populations (endotypes) of patients with bronchiectasis, thereby allowing for more effective studies of new treatments directed at infection, inflammation, and the other aspects of the disease cycle. ${ }^{2,14,20}$

One way to characterize bronchiectasis is the Bronchiectasis Severity Index (BSI), which is a validated tool that incorporates several different patient features to stratify the disease, and thus predict future outcomes such as hospitalization risk and mortality. ${ }^{21}$ A component of the BSI is body mass index (BMI). In the BSI derivation and validation studies, patients with a $B M I<18.5 \mathrm{~kg} /$ $\mathrm{m}^{2}$ had a $31.0 \%$, 4-year mortality rate, which was significantly different compared to patients who were of normal, overweight, and obese BMIs (9.1\%, $7.0 \%$, and $10.4 \%$, 4-year mortality rates, respectively; $p<0.0001){ }^{21}$ This association between mortality and low BMI was preserved when covariates were taken into account, along with prior hospitalizations, 3 or more exacerbations per year, increasing age, and $\mathrm{FEV}_{1} \%$ predicted. ${ }^{21}$ Data from other studies support the notion that nutrition is important in determining outcomes in bronchiectasis. ${ }^{22-25}$ For example, lower BMI appears to be independently associated with radiographic progression of bronchiectasis, as measured by changes in Bhalla scores over a 5-year follow-up period. ${ }^{22}$ Malnutrition (defined as $\mathrm{BMI}<20 \mathrm{~kg} / \mathrm{m}^{2}$ in one study) was shown to be associated with decreased survival in patients with end-stage respiratory disease, including 33 patients with bronchiectasis. ${ }^{23}$ Studies from China suggest that a low BMI is associated with higher mortality, while data from Turkey suggest that better nutrition is beneficial for survival in patients with bronchiectasis. ${ }^{24,25}$ Thus, identifying patient endotypes using nutrition status might help with clinical study stratification. Furthermore, if lower nutrition is indeed associated with worse outcomes, improving nutritional status of patients with bronchiectasis might help improve the disease outlook.

Patient registries are another tool to characterize disease patterns, treatments, and outcomes in a larger number of patients than is possible in single 
center studies. ${ }^{14}$ The U.S. Bronchiectasis and Nontuberculous Mycobacterial Research Registry (U.S. BRR) was created in 2008 for that purpose. ${ }^{9,26}$ Over 2000 patients were enrolled in the BRR as of January 2018, and that number continues to grow. Several studies have been published from the BRR data already to date. ${ }^{27-29}$ Such data provide an opportunity to evaluate nutritional status in a large number of patients with bronchiectasis from across the United States, as well as the ability to compare U.S. patient population data to that published from other countries, as U.S. patients may have different dietary and lifestyle characteristics. ${ }^{9,21,24,25}$

Thus, the goal of this study was to evaluate associations between the nutritional status of the patients enrolled in the U.S. BRR and other markers of bronchiectasis severity. We chose BMI as it has been used to evaluate nutrition in patients with other chronic respiratory diseases, such as chronic obstructive pulmonary disease (COPD). ${ }^{30-32}$ It is an easily obtained and recorded measure for registry purposes, especially when compared to other techniques for measuring adiposity such as skin fold thickness or imaging modalities. ${ }^{21,31,33,34} \mathrm{We}$ hypothesized that a substantial number of patients enrolled in the U.S. BRR are underweight by BMI. We further hypothesized that patientswhoareunderweight have more severe disease (higher frequency of exacerbations and number of hospitalizations, with lower lung function) as compared to patients who were of normal BMI. Finally, we hypothesized that other factors influence both BMI and disease severity, specifically age, gender, smoking status, race/ethnicity, cause of bronchiectasis if known, infection with nontuberculous mycobacteria (NTM) and/or Pseudomonas aeruginosa (P. aeruginosa), the presence or absence of significant gastro-esophageal reflux disease (GERD), and co-existent asthma or COPD. $9,21,35,36$

\section{Methods}

This was a retrospective, observational, longitudinal cohort study using 5-year follow up data from the BRR. The BRR is a database of adult patients ( $\geq 18$ years of age) with physician-established diagnoses of non-CF bronchiectasis and/or NTM lung disease from 15 academic medical centers across the United States. Enrollment of patients was approved by the institutional review board of each study site and for the Data Coordinating Center. Registry data are collected annually from available electronic medical records using standardized data collection forms. ${ }^{9}$ Patients from the BRR were included in this study if they had bronchiectasis confirmed on high resolution CT and had follow-up visit data available over a 5-year time period. Patients were excluded if they had cystic fibrosis, congenital heart disease, inflammatory bowel disease (IBD), human immunodeficiency virus (HIV), a diagnosis of NTM without bronchiectasis, or prior lung transplantation, as we felt these conditions would make nutritional status difficult to interpret.

Based on the baseline visit BMI data, patients were divided into 4 conventional nutrition categories as used by the World Health Organization: underweight $\left(\mathrm{BMI}<18.5 \mathrm{~kg} / \mathrm{m}^{2}\right)$, normal weight $\left(\mathrm{BMI} \geq 18.5 \mathrm{~kg} / \mathrm{m}^{2}\right.$ and $\left.<25.0 \mathrm{~kg} / \mathrm{m}^{2}\right)$, overweight $\left(B M I \geq 25.0 \mathrm{~kg} / \mathrm{m}^{2}\right.$ and $\left.<30.0 \mathrm{~kg} / \mathrm{m}^{2}\right)$, and obese $\left(B M I \geq 30.0 \mathrm{~kg} / \mathrm{m}^{2}\right)$. ${ }^{37} \mathrm{We}$ evaluated the following markers of bronchiectasis disease severity: the number of exacerbations and hospitalizations over the preceding 2 years, pulmonary function tests including forced expiratory volume in 1 second $\left(\mathrm{FEV}_{1}\right)$ and forced vital capacity (FVC) (\% predicted), a history of hemoptysis requiring intervention, and a history of lung resection. Lung function \% predicted values were calculated by each center and internal consistency was present which would counter any discrepancies between different reference equations used at different institutions. Other disease and patient factors were examined using baseline data: age, gender, race/ethnicity, infection with $P$. aeruginosa and/or NTM, smoking status, underlying cause of bronchiectasis, and coexisting diagnoses of GERD, asthma, or COPD. The majority of these characteristics were obtained from the baseline visit data collection forms. Infection with NTM was determined by having either 1 positive sputum culture growing any NTM species at the baseline visit, or if the patient reported a prior diagnosis of NTM infection. The data collection form specifically queried Mycobacterium avium complex (MAC), Mycobacterium abscessus, and Mycobacterium kansasii, with the option to writein additional species under "other." P. aeruginosa infection was measured as having 1 or more positive sputum cultures at baseline.

We then categorized patients by whether their BMI category at subsequent visits remained stable 
(within the same BMI category), had increased to a higher category, or had decreased to a lower category over the 5-year follow-up period. We also evaluated whether patients had an increase in BMI by $>2 \mathrm{~kg} / \mathrm{m}^{2}$, a decrease in BMI by $>2 \mathrm{~kg} / \mathrm{m}^{2}$, or a stable BMI over the 5 -year follow-up period.

\section{Statistical Analysis}

Descriptive statistics were calculated for the overall study sample and stratified by the baseline BMI categories. All results were reported as frequencies and proportions for categorical variables, and as means ( \pm standard deviation [SD]) for continuous variables. Values of the demographic and clinical characteristics were compared between the BMI groups using analysis of variance (ANOVA) for continuous variables, and Chi-square/Fisher exact tests for categorical variables. Post-hoc comparison of adjusted standardized residuals was used to determine the source of the statistically significant Chi-square tests for categorical variables. Posthoc analyses using Dunn's tests were used after statistically significant ANOVAs for continuous variables.

A negative binomial regression model was fit for frequency of exacerbations in the past 2 years prior to enrollment among participants with stable BMI category over time, adjusted for age, gender, presence of Pseudomonas and NTM infection.

Multinomial logistic regression model was fit for the baseline BMI categories adjusted for age, gender, race, history of smoking, GERD, COPD, asthma, baseline $\mathrm{FEV}_{1} \%$ predicted, and NTM. The variable selection was driven by clinical importance and significance of the association with the outcome in the unadjusted analyses.

Missing data analyses compared select demographic and clinical characteristics between the participants who had 5 years of follow-up data and that were included in the analyses, and those participants with incomplete data and that were hence excluded from the analyses. The significance level for all analyses was set at 0.05. The analyses were performed using SAS v.9.4 (Cary, North Carolina) and SPSS version 25 (Armonk, New York).

\section{Results}

Overall, 2461 patients were enrolled in the U.S. BRR at the start of the study in January 2018 (Figure 1). After application of exclusion criteria, this study included 2307 patients, of whom 615 patients had 5 consecutive follow-up visits. Of these, 497 patients had bronchiectasis confirmed on high-resolution CT with either dilated or thickened airways. After excluding 1 patient without a baseline BMI measurement, the final study sample included 496 patients. Table 1 displays baseline demographics of the study population. The majority of patients (83.3\%) were female, and mean age of patients was $65 \pm 13$ years. White patients comprised $92 \%$ of the patient population, while the second largest race represented was Asian patients (5.4\%, n=27). Only 13 patients (2.6\%) identified as black race, and only 12 patients (2.4\%) identified as other race (including multiple races). Very few patients ( $n=15$ in total) identified as Hispanic ethnicity. At the baseline visit, the mean BMI was $22.84 \mathrm{~kg} / \mathrm{m}^{2}(\mathrm{SD}=4.37)$ across all 496 included patients; 61 (12.3\%) were underweight by BMI, 317 (63.9\%) were of normal weight, 86 (17.3\%) were overweight, and 32 (6.5\%) were obese. A greater proportion of men were represented in the overweight and obese groups compared to underweight and normal BMI categories. The patients' age did not significantly differ among BMI groups. A significantly larger percentage of the small number of Asian patients were in the underweight BMI category (16.4\% of all underweight patients, $p=0.003)$. The underweight group of patients had significantly lower lung function than the other weight groups (FVC \% predicted $68.8 \% \pm 21.1 \%$, $p<0.0001$ and $\mathrm{FEV}_{1} \%$ predicted $64.5 \% \pm 22.18 \%$, $p=0.02$ ) (Table 2), however no significant differences were measured among the different BMI groups with regards to exacerbation frequency, hospitalizations, or lung resections.

Other patient characteristics postulated to affect nutrition and thus disease severity were compared across BMI categories at baseline, including infection with NTM and/or P. aeruginosa, underlying cause of bronchiectasis (including primary ciliary dyskinesia [PCD], alpha-1 antitrypsin deficiency [AATD], rheumatological disease, and immunodeficiency), smoking status, and a diagnosis of GERD, COPD, and/or asthma. No significant difference existed in 
Figure 1. Inclusion and Exclusion Criteria of Patients in Study Population

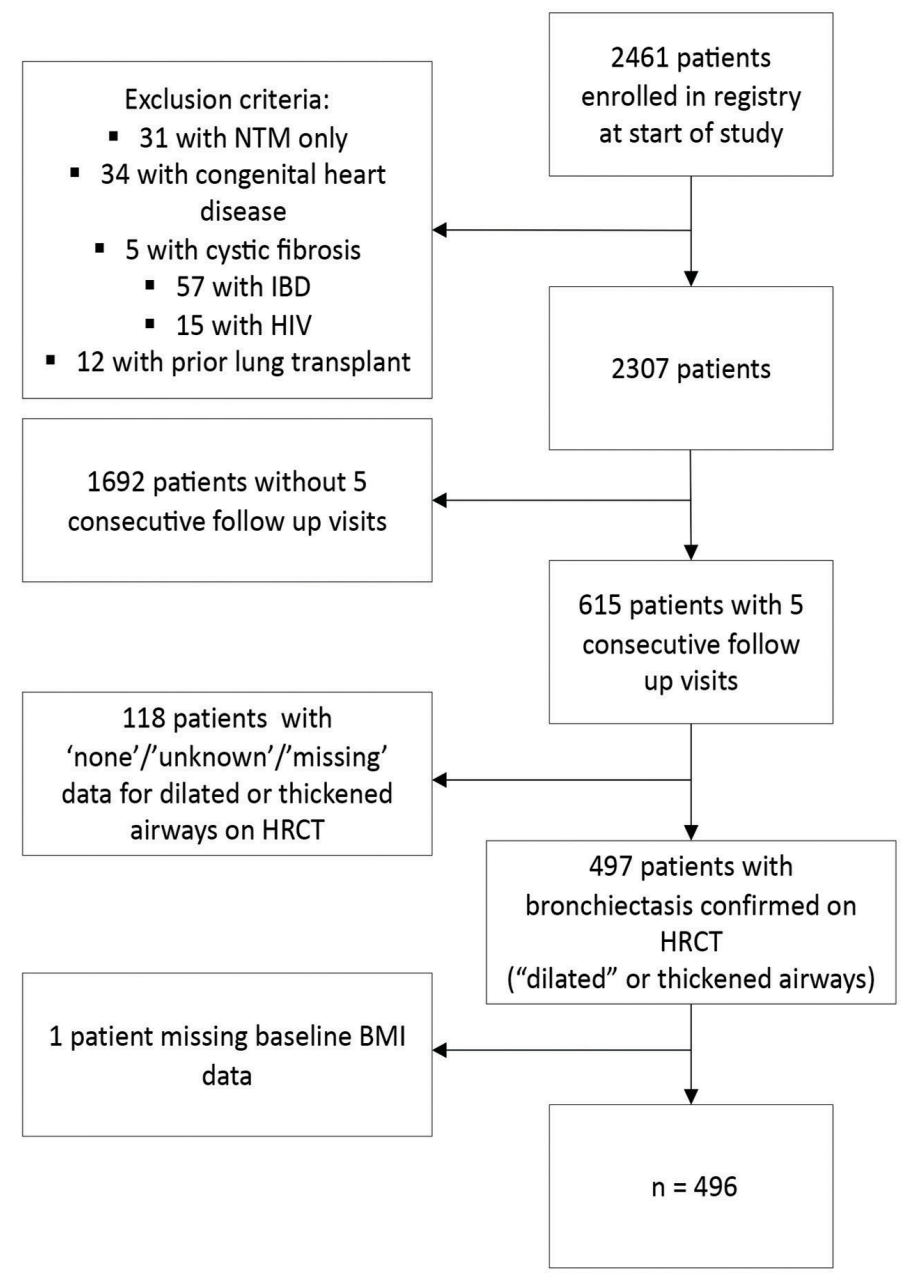

NTM= nontuberculous Mycobacterial; IBD=inflammatory bowel disease; $\mathrm{HRCT}=$ high-resolution computed tomography; $\mathrm{BMI}=$ body mass index

baseline BMI distribution between patients with and without $P$. aeruginosa, as well as between patients with and without NTM (Table 3). Rates of known causes of bronchiectasis, including PCD ( $n=14)$, $\operatorname{AATD}(n=10)$, known rheumatological disease $(n=30)$, and immunodeficiency ( $n=24)$, were not significantly different across all BMI groups, although numberswere relatively small. A significantly higher proportion of obese patients (75\%) carried a diagnosis of GERD as compared to other BMI categories ( $p=0.0015)$ (Table 3). A significantly lower percentage of underweight patients $(11 \%$ ) had been diagnosed with asthma compared to patients in other BMI categories $(p=0.02)$. A greater proportion of patients carrying a diagnosis of COPD and those reporting smoking history were observed as BMI category increased (although not statistically significant, $p=0.06$ and $p=0.153$ respectively).

Of the 496 patients, 406 participants had BMI followup data available over the 5-year period. Analysis of missing data did not reveal significant differences between patients with missing longitudinal BMI data compared to those with complete BMI data (Supplemental Table S1). At the end of the 5-year period, the majority of patients $(n=347,85.5 \%)$ had a stable BMI category, whereas $5.9 \%$ of patients $(n=24)$ had increased their BMI category, and 8.6\% ( $n=35)$ had decreased their BMI category. Evaluation by absolute change in BMI at the end of the 5 -year period also revealed that most patients ( $\mathrm{n}=347,85.5 \%)$ had a stable BMI, whereas $5.4 \%$ of patients $(n=22)$ had a BMI that increased by $>2 \mathrm{~kg} / \mathrm{m}^{2}$, and $9.1 \%$ of patients $(\mathrm{n}=37)$ had a BMI that decreased by $>2 \mathrm{~kg} / \mathrm{m}^{2}$. Given these small numbers, further statistical analyses related to disease outcomes were not performed.

The results of the multivariable regression analyses showed that when controlling for age, race, baseline $\mathrm{FEV}_{1} \%$ predicted, smoking history, and history of GERD, COPD, and asthma, female patients had 3.19 times the odds to have low BMI compared to male patients (95\% CI:1.87-5.45). Similarly, Asian patients had 4.78 times the odds for low BMI compared to white patients (95\% CI: 1.69-13.55) adjusting for age, gender, baseline $\mathrm{FEV}_{1} \%$ predicted, smoking history, and history of GERD, COPD, and asthma. The ordinal logistic regression model showed adequate fit to the data on the score test for the proportional odds assumption $\left(x^{2}(D F=22)=29.79, p=0.12\right)$.

The results of the negative binomial regression did not reveal significant associations between number of exacerbations in the past 2 years prior to enrollment and BMI category $(p=0.30)$, age $(p=0.24)$, gender $(p=0.29)$, or NTM status among the patients with stable BMI. Significant positive association was found between number of exacerbations in the past 2 years and presence of $P$. aeruginosa: IRR $=1.51$ (95\% CI: 1.11-2.04), $p<0.01$. The criteria for assessing goodness of fit showed adequate fit of the model to the data using deviance (scaled deviance) value/DF and Pearson Chi-Square (Scaled Pearson) value/DF. 


\section{Table 1. Select Demographic Characteristics of the Study Population, Overall and by Body Mass Index Categories ${ }^{a}$}

\begin{tabular}{|c|c|c|c|c|c|c|}
\hline & $\begin{array}{l}\text { Overall Sample } \\
\quad N=496\end{array}$ & $\begin{array}{c}\text { Underweight } \\
\qquad \begin{array}{c}\mathrm{N}=61 \\
(12.30 \%)\end{array}\end{array}$ & $\begin{array}{c}\text { Normal } \\
\text { N=317 } \\
(63.91 \%)\end{array}$ & $\begin{array}{l}\text { Overweight } \\
\qquad \begin{array}{c}\mathrm{N}=86 \\
(17.34 \%)\end{array}\end{array}$ & $\begin{array}{c}\text { Obese } \\
N=32 \\
(6.45 \%)\end{array}$ & $p$-Value \\
\hline Age in years Mean (SD) & $64.6(13.3)$ & $65.1(15.9)$ & $64.7(13.0)$ & $64.1(12.9)$ & $64.0(12.8)$ & 0.742 \\
\hline \multicolumn{7}{|l|}{ Gender, $\mathrm{n}(\%)$} \\
\hline Female & $413(83.3)$ & 55 (90.2) & $276(87.1)$ & $64(74.4)$ & $18(56.3)$ & $<.0001$ \\
\hline Male & $83(16.7)$ & $6(9.8)$ & $41(12.9)$ & $22(25.6)$ & $14(43.8)$ & \\
\hline \multicolumn{7}{|l|}{ Race, n (\%) } \\
\hline White & 456 (91.9) & $48(78.7)$ & $300(94.6)$ & 79 (91.9) & 29 (90.6) & 0.001 \\
\hline Black & $13(2.6)$ & $1(1.6)$ & $8(2.5)$ & $2(2.3)$ & $2(6.3)$ & 0.563 \\
\hline Asian & $27(5.4)$ & $10(16.4)$ & $13(4.1)$ & $4(4.7)$ & -- & 0.003 \\
\hline Other (including multiple races) & $12(2.4)$ & $2(3.3)$ & $6(1.9)$ & $3(3.5)$ & $1(3.1)$ & 0.041 \\
\hline $\begin{array}{l}\text { Hispanic Ethnicity, } n(\%) \\
\text { Data available } n=465\end{array}$ & $15(3.2)$ & -- & $8(2.7)$ & $5(6.2)$ & $2(6.5)$ & 0.142 \\
\hline
\end{tabular}

$a_{n=496}$

$\mathrm{SD}=$ standard deviation

\section{Discussion}

As bronchiectasis has been increasingly diagnosed in recent years, it has become important to understand the endotypes within this very heterogeneous population. $3,10,14,20$ One approach is to identify sub-groups of patients of varying nutritional status. Nutrition has been associated with outcomes in other pulmonary diseases including cystic fibrosis as well as COPD. ${ }^{31,34,38}$ Low BMI has previously been linked to increased mortality in patients with bronchiectasis in Europe, China, and Turkey. ${ }^{21,24,25}$ We specifically looked at BMI in the U.S. BRR patient population to further elucidate the role of nutrition in bronchiectasis disease severity. Patients were only included if they had bronchiectasis confirmed on high resolution $\mathrm{CT}$, as this has been acknowledged as the gold standard for diagnosis. ${ }^{39-41}$ As nutritional status may vary over time, we included patients with at least 5 years of consecutive data available in the registry; this study surprisingly revealed that the majority of examined patients maintained a stable BMI over this period. We recognize that excluding patients without 5 years of data limited the number of patients we could include in the study, but we felt it important to structure the study this way as we were interested in not just nutritional status at baseline, but also in nutrition trends; specifically, any change in nutritional status over time.
We hypothesized that there would be a substantial number of patients enrolled in the U.S. BRR who are underweight as measured by BMI. This Lady Windermere description is often noted as the common patient phenotype. ${ }^{42}$ Interestingly and surprisingly, fewer patients than expected were underweight, and the majority of patients (63.9\%) were of normal BMI with a mean BMI of $22.84 \mathrm{~kg} / \mathrm{m}^{2}(\mathrm{SD}=4.37)$ for the study population as a whole. This mean BMI was slightly lower than the mean BMI of the overall BRR cohort at time of analysis $\left(23.5 \mathrm{~kg} / \mathrm{m}^{2}, \mathrm{SD}=5.69\right)$, but both means fell within the normal BMI range. The mean BMI of patients enrolled in the BRR at the time of the "First Look at the U.S. BRR" study publication was also similar, at $23.2 \mathrm{~kg} / \mathrm{m}^{2}(\mathrm{SD}=5.7) .{ }^{9}$ The BMI distribution of our study population is still different from the average U.S. population. In the United States, about a third of older adults (age $\geq 65$ years old) were estimated to be obese by BMI according to 2007-2010 National Health and Nutrition Examination Survey data, compared to $6.5 \%$ of patients in our study population (Table 1 ). ${ }^{43}$ We also hypothesized that being underweight by BMI would be associated with markers of increased disease severity. Indeed, patients who were underweight did have lower lung function than normally nourished or overweight patients, as has been noted before. ${ }^{24}$ The association of low BMI and specifically low fat-free mass in disease severity and mortality in COPD has 


\section{Table 2. Clinical Outcomes and Markers of Disease Severity Measured at Baseline by Body Mass Index Category}

\begin{tabular}{|c|c|c|c|c|c|c|}
\hline & $\begin{array}{l}\text { Overall Sample } \\
\quad N=496\end{array}$ & $\begin{array}{l}\text { Underweight } \\
\qquad \begin{array}{c}\mathrm{N}=61 \\
(12.30 \%)\end{array}\end{array}$ & $\begin{array}{l}\text { Normal } \\
\mathrm{N}=317 \\
(63.91 \%)\end{array}$ & $\begin{array}{l}\text { Overweight } \\
\qquad \begin{array}{c}\mathrm{N}=86 \\
(17.34 \%)\end{array}\end{array}$ & $\begin{array}{c}\text { Obese } \\
N=32 \\
(6.45 \%)\end{array}$ & $p$-Value \\
\hline $\begin{array}{l}\text { Any pulmonary exacerbations } \\
\text { in the past } 2 \text { years, } n(\%) \\
\text { Data available } n=441\end{array}$ & 276 (62.6) & 29 (55.8) & $186(64.4)$ & $42(59.2)$ & $19(65.5)$ & 0.699 \\
\hline $\begin{array}{l}\text { Hospitalizations for pulmonary } \\
\text { illness or exacerbation in the } \\
\text { past } 2 \text { years, } n(\%) \\
\text { Data available } n=441\end{array}$ & 85 (19.3) & 13 (25.0) & 55 (19.0) & 13 (18.3) & $4(13.8)$ & 0.249 \\
\hline $\begin{array}{l}\text { Number of pulmonary } \\
\text { exacerbations in the past } 2 \\
\text { years, Mean (SD) } \\
\text { Data available } n=415\end{array}$ & $1.62(2.2)$ & $1.16(1.4)$ & $1.68(2.3)$ & $1.74(2.6)$ & $1.70(2.1)$ & 0.640 \\
\hline $\begin{array}{l}\text { Number of hospitalizations in } \\
\text { the past } 2 \text { years, Mean (SD) } \\
\text { Data available } n=437\end{array}$ & $0.30(0.8)$ & $0.37(0.7)$ & $0.25(0.6)$ & $0.44(1.5)$ & $0.28(0.8)$ & 0.583 \\
\hline $\begin{array}{l}\text { Pre-Bronchodilator: } \text { FEV }_{\mathbf{1}} \% \\
\text { predicted, Mean (SD) } \\
\text { Data available } \mathrm{n}=448\end{array}$ & $71.87(21.1)$ & $64.49(22.2)$ & $73.54(20.8)$ & $68.54(20.1)$ & 76.48 (21.3) & 0.019 \\
\hline $\begin{array}{l}\text { Pre-Bronchodilator: FVC } \% \\
\text { predicted, Mean (SD) } \\
\text { Data available } n=442\end{array}$ & 80.79 (18.6) & $68.76(21.1)$ & $83.34(16.9)$ & 78.82 (20.3) & $82.03(17.4)$ & $<.0001$ \\
\hline $\begin{array}{l}\text { FEV }_{\mathbf{1}} \mathbf{( L )} / \mathbf{F V C}(\mathbf{L}) \text {, Mean }(\mathrm{SD}) \\
\text { Data available } \mathrm{n}=444\end{array}$ & $0.68(0.1)$ & $0.72(0.1)$ & $0.68(0.1)$ & $0.66(0.1)$ & $0.70(0.1)$ & 0.025 \\
\hline
\end{tabular}

$\mathrm{SD}=$ standard deviation; $\mathrm{FEV}_{1}=$ forced expiratory volume in 1 second; $\mathrm{FVC}=$ forced vital capacity

been described in the literature, but the underlying mechanics have not been fully elucidated. ${ }^{31,44}$ Loss of muscle mass in patients with lower nutritional status (specifically, fat-free mass index) has been hypothesized to contribute to worsening of lung function in patients with COPD; increased metabolic demand from poor respiratory function has also been theorized to contribute to low weight and muscle mass in this patient population. ${ }^{34,44-46}$ Potentially, these mechanisms are also active in underweight patients with bronchiectasis. ${ }^{32}$

Despite this association of lower BMI with lower lung function, lower BMI did not appear to be associated with what are considered the main markers of disease severity and those that are considered to affect disease prognosis: hospitalization rates and frequency of exacerbations. Of note, the frequency of exacerbations in our subgroup was lower than the frequency of exacerbations over the preceding
2 years that was reported in the U.S. BRR "First Look" paper (1.62 \pm 2.2 , compared with $3.0 \pm 2.7) .{ }^{9}$ We may have not detected an association between BMI and exacerbations because of low frequency of exacerbations in this subgroup, or perhaps the subgroup in this study was somehow different than the larger group in the U.S. BRR. We discuss some limitations of the study relevant to this observation (including possible selection bias) below.

There are few other published data in regards to BMI and hospitalizations. One study from China ( $\mathrm{n}=339$ patients) did show that BMI was an independent predictor of hospitalization; that is, underweight patients with bronchiectasis had increased risk of hospitalization. ${ }^{24}$ It should be noted that this was a multi-center study of Chinese patients, with somewhat different study population characteristics compared to our current study. For example, there was a slightly higher percentage of underweight patients 


\section{Table 3. Select Comorbidities and Microbiology Results at Baseline by Body Mass Index Category}

\begin{tabular}{|c|c|c|c|c|c|c|}
\hline & $\begin{array}{l}\text { Overall Sample } \\
\qquad N=496\end{array}$ & $\begin{array}{l}\text { Underweight } \\
\qquad \begin{array}{c}\mathrm{N}=61 \\
(12.30 \%)\end{array}\end{array}$ & $\begin{array}{c}\text { Normal } \\
\mathrm{N}=317 \\
(63.91 \%)\end{array}$ & $\begin{array}{l}\text { Overweight } \\
\qquad \begin{array}{c}\mathrm{N}=86 \\
(17.34 \%)\end{array}\end{array}$ & $\begin{array}{l}\text { Obese } \\
\mathrm{N}=32 \\
(6.45 \%)\end{array}$ & $p$-Value \\
\hline $\begin{array}{l}\text { Pseudomonas aeruginosa }{ }^{a} \\
\mathrm{n}(\%) \text { Data available } \mathrm{n}=350\end{array}$ & $124(35.4)$ & 11 (29.7) & 86 (37.6) & 20 (32.8) & $7(30.4)$ & 0.732 \\
\hline $\begin{array}{l}\text { NTM, }{ }^{\mathrm{b}} \mathrm{n}(\%) \\
\text { Data available } \mathrm{n}=496\end{array}$ & 271 (54.6) & $34(55.7)$ & $180(56.8)$ & 46 (53.5) & $11(34.4)$ & 0.068 \\
\hline $\begin{array}{l}\text { Ever diagnosed with GERD, } \\
\mathrm{n}(\%) \text { Data available } \mathrm{n}=485\end{array}$ & $235(48.5)$ & $24(40.7)$ & $142(45.9)$ & 45 (52.9) & 24 (75.0) & 0.002 \\
\hline $\begin{array}{l}\text { Ever diagnosed with COPD, } \\
\mathrm{n}(\%) \text { Data available } \mathrm{n}=460\end{array}$ & $92(20.0)$ & $8(14.6)$ & $57(19.1)$ & $18(23.4)$ & $9(30.0)$ & 0.060 \\
\hline $\begin{array}{l}\text { Ever diagnosed with asthma, } \\
\mathrm{n}(\%) \text { Data available } \mathrm{n}=463\end{array}$ & $123(26.6)$ & $6(10.7)$ & 84 (27.9) & $23(30.3)$ & $10(33.3)$ & 0.021 \\
\hline $\begin{array}{l}\text { Current or former smoker, } \\
n(\%) \text { Data available } n=463\end{array}$ & $181(39.1)$ & $17(29.8)$ & 115 (38.3) & 33 (43.4) & 16 (53.3) & 0.153 \\
\hline $\begin{array}{l}\text { Ever diagnosed with AATD, } \\
\mathrm{n}(\%) \text { Data available } n=466\end{array}$ & $10(2.2)$ & $2(3.5)$ & $6(2.0)$ & -- & $2(6.7)$ & 0.127 \\
\hline $\begin{array}{l}\text { Ever diagnosed with primary } \\
\text { immunodeficiency, } n(\%) \\
\text { Data available } n=465\end{array}$ & $24(5.2)$ & $1(1.8)$ & $17(5.7)$ & $3(3.9)$ & $3(10.0)$ & 0.128 \\
\hline $\begin{array}{l}\text { Ever diagnosed with } \\
\text { Kartagener's } \\
\text { syndrome or PCD, n (\%) } \\
\text { Data available } n=466\end{array}$ & $14(3.0)$ & -- & 11 (3.6) & $3(3.9)$ & -- & 0.361 \\
\hline $\begin{array}{l}\text { Ever diagnosed with a } \\
\text { rheumatologic disease, }{ }^{\mathrm{C}} \mathrm{n}(\%) \\
\text { Data available } \mathrm{n}=466\end{array}$ & $30(6.4)$ & $2(3.5)$ & $19(6.3)$ & 7 (9.01) & $2(6.7)$ & 0.076 \\
\hline
\end{tabular}

${ }^{a}$ Defined as one or more positive cultures at baseline

${ }^{b}$ NTM $(+)$ patients defined as those with either a reported history of pulmonary NTM disease and/or those with one or more NTM isolates in respiratory specimen cultures at baseline

${ }^{\mathrm{c}}$ Rheumatologic disease including rheumatoid arthritis, Sjogren's syndrome

$\mathrm{NTM}=$ non-tuberculous mycobacteria; GERD=gastro-esophageal reflux disease; $\mathrm{COPD}=$ chronic obstructive pulmonary disease; AATD=alpha-1 antitrypsin deficiency; PCD=primary ciliary dyskinesia

(29\%) compared to our study population, as well as a higher proportion of male patients ( $42 \%$ versus $17 \%$ in our study), with no significant difference in BMI distribution for male and female patients.

Our study also revealed that Asian race was significantly associated with a lower BMI when controlling for other variables. There has been much debate and controversy over implementing ethnicityspecific BMI parameters to define being overweight and obese, specifically in Asian nations where lower BMIs would be considered overweight or obese as compared to the classifications used in white patients. ${ }^{47}$ One strategy would be to recategorize
Asian patients in our study by ethnicity specific BMI categories, if known. Overall, patients of Asian race from our study comprised a small percentage of our sample size ( $n=27$, or $5.4 \%$ ). Our initial study question stemmed from the hypothesis that the U.S. population may show different trends with relation to BMI compared to other populations that have been studied. These results suggest that the Asian patients with bronchiectasis might be distinctive within the U.S. population, and may explain why the results from our predominantly white study population differ from the results of $\mathrm{Qi}$ et al. ${ }^{24}$

We also hypothesized that other factors would 
influence BMI. Infections clearly play a role in bronchiectasis severity and disease course over time, with several studies showing that chronic infection with $P$. aeruginosa has been associated with increased exacerbation frequency and hospitalizations, and decreased quality of life. ${ }^{35,36,48}$ This study also confirmed the association of infection with $P$. aeruginosa and increased exacerbation frequency in patients with stable BMI. However, despite the clinical importance of Pseudomonas infection, the data from this study did not show any relationship between the presence of Pseudomonas and nutrition status.

In addition to $P$. aeruginosa, mycobacterial infection is a common problem in patients with bronchiectasis. $9,49,50$ Studies have shown that in patients with NTM, having a lower BMI was associated with increased clinical deterioration and radiographic progression. ${ }^{51,52}$ Our study did not, however, show any differences in BMI distribution between patients with and without NTM. That is to say, the majority of patients fell within the normal BMI category regardless of NTM status (defined as either a reported history of pulmonary NTM disease and/ or one or more NTM isolates in respiratory specimen cultures at baseline, which may not reflect clinically significant NTM infection). The "First Look at the U.S. BRR" study reported that patients with bronchiectasis and NTM had lower BMIs on average compared to other patients with bronchiectasis without NTM, although both groups did fall within the normal BMI range. ${ }^{9}$ Thus, using BMI categorization rather than absolute BMI number may account for why our study did not appreciate a statistically significant difference between patients with NTM and patients without NTM.

Compared to the stereotype of the tall, thin, female patient with bronchiectasis, there were more male patients represented in the overweight and obese categories. In addition to Asian race, cumulative odds regression analysis revealed that female sex was also significantly associated with low BMI when controlling for other variables.

As alluded to previously, in addition to looking at the cross-sectional data, we examined the longitudinal BMI data from those patients with at least 5 years of follow-up visits. We postulated that patients with a decline in BMI (as defined by a change in BMI category - for example, from normal to underweight; and separately defined by an absolute change in BMI by an increase or decrease in $>2 \mathrm{~kg} / \mathrm{m}^{2}$ ) would have increased markers of disease severity as compared to patients whose BMI remained stable, or probably less commonly, increased. Surprisingly, our data revealed that, for the majority of patients, BMI remained stable by absolute BMI number and by BMI category. This suggests that either patients with bronchiectasis have a more stable nutritional status that one might expect, or that a longer period of study and/or larger sample size may be required to see differences in nutritional status and disease outcome measures. The small subset of patients that did have a decline in weight may represent an important sub-endotype within bronchiectasis, warranting further evaluation with larger numbers of patients.

Limitations of the study include the challenge of incomplete data in the setting of a retrospective longitudinal study, which limited the sample size. We attempted to compare patients who had missing data to those who had complete data, to verify that there was not a systematic issue of attrition bias, bearing in mind that limitations of the data collection methodology means that the exact reason that data were missing is unknown. As previously noted, restricting the patient population to only those with at least 5 years of follow up data rather than all patients with baseline data did limit the number of patients we included in the study and may have introduced selection bias; however, for this initial study we wanted to evaluate the change in nutritional status over time. The potential of selection bias remains, as our subgroup may have differences from the larger U.S. BRR study population given differences seen in exacerbation frequency between the 2 groups. ${ }^{9}$ As documented deaths were low in this study, we are not able to draw conclusions regarding mortality, and our retrospective longitudinal study approach was not intended to examine this question. As data were obtained from patients from tertiary institutions, these results may not be generalizable. While multicenter studies allow for larger study populations to be enrolled, the potential for heterogeneity of practices between institutions exists. Another limitation which had the potential to influence the findings is the exclusion of patients with IBD; however, they comprised a small portion of the registry population (3\%) as a whole and a similarly small number in our study population. 
As the bronchiectasis community continues to learn more about the disease and patient endo/ phenotypes, further elucidation of the mechanism and pathophysiology of disease outcomes and their interrelatedness is important, especially in the context of the development of new therapies. BMI is only one surrogate for describing a patient's complex state of nutrition. It does not account for body shape variations and composition (such as muscle mass and adipose tissue distribution). ${ }^{33}$ The loss of muscle mass, rather than loss of fat, has been implicated as the driver of worse outcomes in patients with COPD. ${ }^{34,45,46}$ Thus, future studies may need to address other measures to better define nutritional status and body composition, such as bioelectrical impedance, dual-energy X-ray absorptiometry, $\mathrm{CT}$, and magnetic resonance imaging that can describe fat-free mass. ${ }^{33,53}$ For example, fat-free mass is one measure which was found to better correlate with nutritional status in a subset of patients with bronchiectasis requiring long-term oxygen. ${ }^{32}$ C-reactive protein and other markers of inflammation are also of interest as far as interplay between nutritional status and disease outcomes in respiratory illness, including bronchiectasis. ${ }^{23}$ These remain important avenues of investigation, as ultimately, among other therapies, nutritional interventions might offer opportunities for benefit in the disease over time.

Future directions for study, which have the potential to overcome some of the limitations of this initial registry study, include longer followup intervals, larger sample sizes, and broadening patient inclusion criteria to incorporate patients with lung transplantation or other underlying conditions such as IBD. Additionally, as the U.S. BRR continues to grow and as, for example, more data on patients with NTM lung disease become available, it should prove advantageous to re-examine the role of NTM infection and nutrition. Ideally, one would like to evaluate patients who met American Thoracic Society criteria for NTM requiring treatment. Therapies for NTM may have a variety of side effects, including gastrointestinal symptoms which potentially have an impact on nutritional status. ${ }^{54}$ Another direction for future study would be to evaluate rates of Pseudomonas acquisition from baseline over time in patients with declining weight compared to those with stable or increasing weight. Studies using more advanced methodology to evaluate nutritional status should be quite informative. Ongoing investigation of nutrition in European populations, building on the role of BMI as part of the BSI, and other populations across the globe may help elucidate suggested differences in nutritional status by race.

In conclusion, this study shows that the majority of patients with bronchiectasis in the United States are of normal BMI. Although underweight patients with bronchiectasis have lower lung function than normally nourished or overweight patients, lower BMI does not appear to relate to other markers of disease severity in this patient population. Gender and race seem to independently influence nutritional status of patients with bronchiectasis. Larger sample sizes and longer study periods, along with more refined techniques to measure nutritional status and body composition, are needed to further evaluate the role of nutrition in bronchiectasis.

\section{Acknowledgements}

The authors would like to acknowledge the COPD Foundation, a 501(c)(3) nonprofit organization, who manages the Bronchiectasis and NTM Research Registry. The Registry is funded by the Richard $\mathrm{H}$. Scarborough Bronchiectasis Research Fund, the Anna-Maria and Stephen Kellen Foundation, and the Bronchiectasis and NTM Industry Advisory Committee. It should also be noted that this work would not have been possible without the comprehensive chart reviews and recording of data by the dedicated research coordinators and principal investigators at each of the participating Registry sites. The authors would also like to acknowledge the staff of the COPD Foundation for their commitment to the success of the Registry. We acknowledge the work of Vira Pravosud who assisted in early data analysis for this project. We acknowledge the editorial assistance of the NC Translational and Clinical Sciences Institute, which is supported by the National Center for Advancing Translational Sciences, National Institutes of Health, through grant award number UL1TR002489.

Author contributions: KAD, RC, LAD, and PGN had full access to all the data in the study and take full responsibility for the integrity of the data and the accuracy of the data analysis. MRK significantly contributed to the study design and contributed to the paper by review and comments. D A-H, TRA, AB, AB, 
CLD, EE, AD, KF, JP, MMJ, PJM, MLM, AEO, KNO, MAS, AS, BT, GT, and KLW substantially contributed to the paper by review, comments, and contribution to the dataset. All authors significantly contributed to the intellectual content of the

article.

\section{Declaration of Interest}

Dr. Addrizzo-Harris reports consultant work from Insmed, advisory board work from AIT, outside the submitted work. Dr. Basavaraj reports grants from the COPD Foundation, personal fees from Hill-Rom, and personal fees from Insmed, outside the submitted work. Dr. Daley reports grants from the COPD Foundation, during the conduct of the study and grants from Insmed, outside the submitted work. Dr. Daniels reports grants and personal fees from Insmed, personal fees from Spark Healthcare, personal fees from International Biophysics Corporation, grants from Zambon, and grants from Parion/Vertex, outside the submitted work. Dr. Noone has received grant support from Aradigm/Grifols, Insmed, Parion/ Vertex and Bayer, and consultancy fees from Bayer, Grifols, and Smartvest. Dr. O’Donnell reports grants from the COPD Foundation/U.S. Bronchiectasis Research Registry, grants and personal fees from Insmed Inc, personal fees from Electromed and
Merck, grants and personal fees from Bayer, personal fees from Xellia, and grants from Zambon, Aradigm, and Parion, outside the submitted work. Dr. Olivier reports grants from Beyond Air, Inc, and Matinas Biopharma, outside the submitted work. Dr. Philley reports personal fees from Insmed, Bayer, and Janssen, grants from REPORT trial, outside the submitted work. Dr. Tino reports grants from the U.S. BRR/COPD Foundation, advisory board work from Bayer, Grifols, Aradigm and Cipla, outside the submitted work. Dr. Salathe reports grants from the COPD Foundation (registry), during the conduct of the study; grants and personal fees from the National Institutes of Health, the Flight Attendant Medical Research Institute, and Arrowhead Pharmaceuticals and grants from the James and Esther King Florida Biomedical Research Program, outside the submitted work. Dr. Thomashow reports personal fees from GSK and Astra Zeneca, outside the submitted work. Dr. Winthrop reports grants and personal fees from Insmed, personal fees from Johnson \& Johnson, Paratek, Red Hill Biopharma, and Horizon, outside the submitted work. Drs. Aksamit, Barker, Despotes, Choate, DiMango, Eden, Fennelly, Johnson, Knowles, McShane, Metersky, and Schmid have no conflicts of interest to disclose. 


\section{References}

1. Chalmers JD, Chang AB, Chotirmall SH, Dhar R, McShane PJ. Bronchiectasis. Nat Rev Dis Primers. 2018;4(1):45.

doi: https://doi.org/10.1038/s41572-018-0042-3

2. Henkle E, Aksamit TR, Daley CL, et al. US Patient-centered research priorities and roadmap for bronchiectasis. Chest. 2018;154(5):10161023. doi: https://doi.org/10.1016/j.chest.2018.06.032

3. Weycker D, Hansen GL, Seifer FD. Prevalence and incidence of noncystic fibrosis bronchiectasis among US adults in 2013. Chron Respir Dis. 2017;14(4):377-384.

doi: https://doi.org/10.1177/1479972317709649

4. O'Leary CJ, Wilson CB, Hansell DM, Cole PJ, Wilson R, Jones PW. Relationship between psychological well-being and lung health status in patients with bronchiectasis. Respir Med. 2002;96(9):686-692. doi: https://doi.org/10.1053/rmed.2002.1330

5. Hill AT, Haworth CS, Aliberti S, et al. Pulmonary exacerbation in adults with bronchiectasis: a consensus definition for clinical research. Eur Respir J. 2017;49(6):170051.

doi: https://doi.org/ 10.1183/13993003.00051-2017

6. Chalmers JD, Aliberti S, Blasi F. Management of bronchiectasis in adults. Eur Respir J. 2015;45(5):1446-1462.

doi: https://doi.org/10.1183/09031936.00119114

7. Hill AT, Welham SA, Sullivan AL, Loebinger MR. Updated BTS Adult Bronchiectasis Guideline 2018: a multidisciplinary approach to comprehensive care. Thorax. 2019;74(1):1-3.

doi: https://doi.org/10.1136/thoraxjnl-2018-212468

8. Polverino E, Goeminne PC, McDonnell MJ, et al. European Respiratory Society guidelines for the management of adult bronchiectasis. Eur Respir J. 2017;50(3):1700629.

doi: https://doi.org/10.1183/13993003.00629-2017

9. Aksamit TR, O'Donnell AE, Barker A, et al. Adult patients with bronchiectasis: a first look at the US Bronchiectasis Research Registry. Chest. 2017;151(5):982-992.

doi: https://doi.org/10.1016/j.chest.2016.10.055

10. Quint JK, Millett ER, Joshi M, et al. Changes in the incidence, prevalence and mortality of bronchiectasis in the UK from 2004 to 2013: a population-based cohort study. Eur Respir J. 2016;47(1):186193. doi: https://doi.org/10.1183/13993003.01033-2015

11. McShane PJ, Naureckas ET, Strek ME. Bronchiectasis in a diverse US population: effects of ethnicity on etiology and sputum culture. Chest. 2012;142(1):159-167.

doi: https://doi.org/10.1378/chest.11-1024

12. Lonni S, Chalmers JD, Goeminne PC, et al. Etiology of non-cystic fibrosis bronchiectasis in adults and its correlation to disease severity. Ann Am Thorac Soc. 2015;12(12):1764-1770. doi: https://doi.org/10.1513/AnnalsATS.201507-472OC
13. Seitz AE, Olivier KN, Adjemian J, Holland SM, Prevots DR. Trends in bronchiectasis among medicare beneficiaries in the United States, 2000 to 2007. Chest. 2012;142(2):432-439. doi: https://doi.org/10.1378/chest.11-2209

14. Chalmers JD, Aliberti S, Polverino E, et al. The EMBARC European Bronchiectasis Registry: protocol for an international observational study. ERJ Open Res. 2016;2(1):00081-2015. doi: https://doi.org/10.1183/23120541.00081-2015

15. De Soyza A, Aksamit T, Bandel TJ, et al. RESPIRE 1: a phase III placebocontrolled randomised trial of ciprofloxacin dry powder for inhalation in non-cystic fibrosis bronchiectasis. Eur Respir J. 2018;51(1):1702052. doi: https://doi.org/10.1183/13993003.02052-2017

16. AksamitT, De Soyza A, BandelTJ, etal. RESPIRE 2: a phase III placebocontrolled randomised trial of ciprofloxacin dry powder for inhalation in non-cystic fibrosis bronchiectasis. Eur Respir J. 2018;51(1):1702053. doi: https://doi.org/10.1183/13993003.02053-2017

17. Aksamit T, Bandel TJ, Criollo M, et al. The RESPIRE trials: two phase III, randomized, multicentre, placebo-controlled trials of ciprofloxacin dry powder for inhalation (Ciprofloxacin DPI) in non-cystic fibrosis bronchiectasis. Contemp Clin Trials. 2017;58:78-85. doi: https://doi.org/10.1016/j.cct.2017.05.007

18. Haworth CS, Bilton D, Chalmers JD, et al. Inhaled liposomal ciprofloxacin in patients with non-cystic fibrosis bronchiectasis and chronic lung infection with Pseudomonas aeruginosa (ORBIT-3 and ORBIT-4): two phase 3, randomised controlled trials. Lancet Respir Med. 2019;7(3):2113-216. doi: https://doi.org/10.1016/S2213-2600(18)30427-2

19. McShane PJ, Tino G. Bronchiectasis. Chest. 2018;155(4):825-833. doi: https://doi.org/10.1016/j.chest.2018.10.027

20. Aliberti S, Masefield S, Polverino E, et al. Research priorities in bronchiectasis: a consensus statement from the EMBARC Clinical Research Collaboration. Eur Respir J. 2016;48(3):632-647. doi: https://doi.org/10.1183/13993003.01888-2015

21. Chalmers JD, Goeminne P, Aliberti S, et al. The bronchiectasis severity index. An international derivation and validation study. Am J Respir Crit Care Med. 2014;189(5):576-585.

doi: https://doi.org/10.1164/rccm.201309-1575OC

22. Park J, Kim S, Lee YJ, et al. Factors associated with radiologic progression of non-cystic fibrosis bronchiectasis during long-term follow-up. Respirology. 2016;21(6):1049-1054. doi: https://doi.org/10.1111/resp.12768

23. Cano NJ PC, Roth H, Court-Fortuné I, et al; Clinical Research Group of the Société Francophone de Nutrition Entérale et Parentérale. $\mathrm{C}$-reactive protein and body mass index predict outcome in end-stage respiratory failure. Chest. 2004;126(2):540-546. doi: https://doi.org/10.1378/chest.126.2.540

24. Qi Q, Li T, Li JC, Li Y. Association of body mass index with disease severity and prognosis in patients with non-cystic fibrosis bronchiectasis. Braz J Med Biol Res. 2015;48(8):715-724. doi: https://doi.org/10.1590/1414-431x20154135 
25. Onen $\mathrm{ZP}$, Gulbay $\mathrm{BE}$, Sen $\mathrm{E}$, et al. Analysis of the factors related to mortality in patients with bronchiectasis. Respir Med. 2007;101(7):1390-1397.

doi: https://doi.org/10.1016/j.rmed.2007.02.002

26. McShane PJ. Bronchiectasis: an orphan finds a home. Chest. 2017;151(5):953-954.

doi: https://doi.org/10.1016/j.chest.2016.12.026

27. Metersky ML, Aksamit TR, Barker A, et al. The prevalence and significance of Staphylococcus aureus in patients with non-cystic fibrosis bronchiectasis. Ann Am Thorac Soc. 2018;15(3):365-370. doi: https://doi.org/10.1513/AnnalsATS.201706-426OC

28. Ruffner MA, Aksamit TR, Thomashow B, et al. Frequency of untreated hypogammaglobulinemia in bronchiectasis. Ann Allergy Asthma Immunol. 2017;119(1):83-85.

doi: https://doi.org/10.1016/j.anai.2017.04.020

29. Henkle E, Aksamit TR, Barker AF, et al. Pharmacotherapy for noncystic fibrosis bronchiectasis: results from an $\mathrm{ntm}$ info \& research patient survey and the bronchiectasis and ntm research registry. Chest. 2017;152(6):1120-1127.

doi: https://doi.org/10.1016/j.chest.2017.04.167

30. Steinkamp G, Wiedemann B. Relationship between nutritional status and lung function in cystic fibrosis: cross sectional and longitudinal analyses from the German $\mathrm{CF}$ quality assurance (CFQA) project. Thorax. 2002;57(7):596-601.

doi: https://doi.org/10.1136/thorax.57.7.596

31. Celli BR, Cote CG, Marin JM, et al. The body-mass index, airflow obstruction, dyspnea, and exercise capacity index in chronic obstructive pulmonary disease. N Engl J Med. 2004;350(10):10051012. doi: https://doi.org/10.1056/NEJMoa021322

32. Cano NJ, Roth H, Court-Ortune I, et al. Nutritional depletion in patients on long-term oxygen therapy and/or home mechanical ventilation. Eur Respir J. 2002;20(1):30-37. doi: https://doi.org/10.1183/09031936.02.01812001

33. Cornier MA, Despres JP, Davis N, et al. Assessing adiposity: a scientific statement from the American Heart Association. Circulation. 2011;124(18):1996-2019.

doi: https://doi.org/10.1161/CIR.0b013e318233bc6a

34. Cao C, Wang R, Wang J, Bunjhoo H, Xu Y, Xiong W. Body mass index and mortality in chronic obstructive pulmonary disease: a metaanalysis. PLoS One. 2012;7(8):e43892.

doi: https://doi.org/10.1371/journal.pone.0043892

35. Araujo D, Shteinberg M, Aliberti S, et al. The independent contribution of Pseudomonas aeruginosa infection to long-term clinical outcomes in bronchiectasis. Eur Respir J. 2018;51(2). doi: https://doi.org/10.1183/13993003.01953-2017
36. Finch S, McDonnell MJ, Abo-Leyah H, Aliberti S, Chalmers JD. A comprehensive analysis of the impact of Pseudomonas aeruginosa colonization on prognosis in adult bronchiectasis. Ann Am Thorac Soc. 2015;12(11):1602-1611.

doi: https://doi.org/10.1513/AnnalsATS.201506-333OC

37. World Health Organization (WHO). Obesity: preventing and managing the global epidemic. Report of a WHO consultation. WHO website. Published 2000. Accessed June 2020. https://www.who.int/ nutrition/publications/obesity/WHO_TRS_894/en/

38. Hallin R, Koivisto-Hursti UK, Lindberg E, Janson C. Nutritional status, dietary energy intake and the risk of exacerbations in patients with chronic obstructive pulmonary disease (COPD). Respir Med. 2006;100(3):561-567.

doi: https://doi.org/10.1016/j.rmed.2005.05.020

39. Grenier P, Maurice F, Musset D, Menu Y, Nahum H. Bronchiectasis: assessment by thin-section CT. Radiology. 1986;161(1):95-99. doi: https://doi.org/10.1148/radiology.161.1.3763889

40. Joharjy IA, Bashi SA, Adbullah AK. Value of medium-thickness CT in the diagnosis of bronchiectasis. AJR Am J Roentgenol. 1987;149(6):1133-1137. doi: https://doi.org/10.2214/ajr.149.6.1133

41. Smith IE, Jurriaans E, Diederich S, Ali N, Shneerson JM, Flower CD. Chronic sputum production: correlations between clinical features and findings on high resolution computed tomographic scanning of the chest. Thorax. 1996;51(9):914-918. doi: https://doi.org/10.1136/thx.51.9.914

42. Olivier KN. Lady Windermere dissected: more form than fastidious. Ann Am Thorac Soc. 2016;13(10):1674-1676. doi: https://doi.org/10.1513/AnnalsATS.201607-521ED

43. Fakhouri TH, Ogden CL, Carroll MD, Kit BK, Flegal KM. Prevalence of obesity among older adults in the United States, 2007-2010. NCHS Data Brief. 2012(106):1-8.

44. Kwan HY, Maddocks M, Nolan CM, et al. The prognostic significance of weight loss in chronic obstructive pulmonary disease-related cachexia: a prospective cohort study. J Cachexia Sarcopenia Muscle. 2019;10(6):1330-1338. doi: https://doi.org/10.1002/jcsm.12463

45. Vestbo J, Prescott E, Almdal T, et al. Body mass, fat-free body mass, and prognosis in patients with chronic obstructive pulmonary disease from a random population sample: findings from the Copenhagen City Heart Study. Am J Respir Crit Care Med. 2006;173(1):79-83. doi: https://doi.org/10.1164/rccm.200506-9690C

46. Ischaki E, Papatheodorou G, Gaki E, Papa I, Koulouris N, Loukides S. Body mass and fat-free mass indices in COPD: relation with variables expressing disease severity. Chest. 2007;132(1):164-169. doi: https://doi.org/10.1378/chest.06-2789

47. Misra A. Ethnic-specific criteria for classification of body mass index: a perspective for Asian Indians and American Diabetes Association Position Statement. Diabetes Technol Ther. 2015;17(9):667-671. doi: https://doi.org/10.1089/dia.2015.0007 
48. Chalmers JD, Aliberti S, Filonenko A, et al. Characterization of the frequent exacerbator phenotype in bronchiectasis. Am J Respir Crit Care Med. 2018;197(11):1410-1420.

doi: https://doi.org/10.1164/rccm.201711-2202OC

49. Sexton P, Harrison AC. Susceptibility to nontuberculous mycobacterial lung disease. Eur Respir J. 2008;31(6):1322-1333.

doi: https://doi.org/10.1183/09031936.00140007

50. Mirsaeidi M, Hadid W, Ericsoussi B, Rodgers D, Sadikot RT. Nontuberculous mycobacterial disease is common in patients with noncystic fibrosis bronchiectasis. Int $J$ Infect Dis. 2013;17(11):e10001004. doi: https://doi.org/10.1016/j.ijid.2013.03.018

51. Kim SJ, Park J, Lee H, et al. Risk factors for deterioration of nodular bronchiectatic Mycobacterium avium complex lung disease. Int $J$ Tuberc Lung Dis. 2014;18(6):730-736.

doi: https://doi.org/10.5588/ijtld.13.0792

52. Pan SW, Shu CC, Feng JY, et al. Microbiological persistence in patients with mycobacterium avium complex lung disease: the predictors and the impact on radiographic progression. Clin Infect Dis. 2017;65(6):927-934. doi: https://doi.org/10.1093/cid/cix479

53. Thibault R, Pichard C. The evaluation of body composition: a useful tool for clinical practice. Ann Nutr Metab. 2012;60(1):6-16. doi: https://doi.org/10.1159/000334879

54. Bedi P, Chalmers JD, Goeminne PC, et al. The BRICS (Bronchiectasis Radiologically Indexed CT Score): a multicenter study score for use in idiopathic and postinfective bronchiectasis. Chest. 2018;153(5):11771186. doi: https://doi.org/10.1016/j.chest.2017.11.033 